\title{
PHILOS Plate: Is it the Panacea for all Proximal Humerus Fractures?
}

\section{Ravi Kumar Gupta*, Anubhav Malhotra, Pawan Kumar and Gladson David Masih}

Department of Orthopaedics, Government Medical College Hospital, Chandigarh, Punjab, India

\begin{abstract}
The problem of fixation of 3 and 4 part displaced proximal humerus fractures was thought to be greatly solved with the evolution of proximal humerus internal locking system (PHILOS). We report a case of migration of screws of PHILOS plate into the shoulder joint, where salvage was done with hemi-arthroplasty. The decision for osteosynthesis in complex 3 and 4 part fracture needs a high index of awareness of the potential complications of PHILOS.
\end{abstract}

\section{Introduction}

Proximal humerus fractures are common and have a bimodal age distribution. Fracture-dislocations in younger patients result from highenergy trauma and most surgeons attempt open reduction and internal fixation, if at all possible. Osteoporotic fractures in elderly patients are commonly associated with low-energy trauma such as groundlevel falls and most are minimally displaced, impacted fractures that can be treated successfully with non-operative means. However, the optimal surgical management of three and four-part proximal humeral fractures in elderly osteoporotic patients remains controversial, with many advocating prosthetic replacement of the humeral head [1]. Recent developments in plating with the use of locking screws offer mechanical advantages for the treatment of displaced fractures of the proximal humerus [2]. Biomechanical data suggest that these implants can resist physiological loads in osteoporotic bone and may provide an alternative to hemi-arthroplasty [3]. Theoretically, these plates provide more secure fixation of proximal humeral fractures, especially in weak or osteoporotic bones [4]. Nevertheless, the associated complication rate remains high (over 10\%) [5]. The rate of avascular necrosis of humeral head after 3- or 4-part fractures ranges between 12-25\% and $41-59 \%$ respectively [6]. Furthermore, the increased number of screws in the humeral head may affect fracture-healing and perfusion of humeral head, thus contributing towards further osteonecrosis of humeral head [2]. Complications, including loss of fixation, humeral head perforation, and mechanical impingement, have been reported [1]. We present a case of failed PHILOS plate in a middle aged woman where osteoporosis and comminuted fracture pattern resulted in migration of locking screws into the shoulder joint, causing further damage to humeral head.

\section{Case Report}

60 -year-old lady, teacher by profession, was referred to us with an extremely painful right shoulder. Five months ago, she had underwent

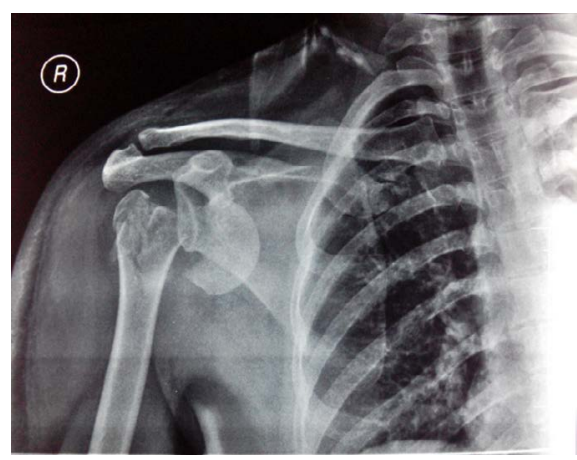

Figure 1: Fracture dislocation of proximal humerus.

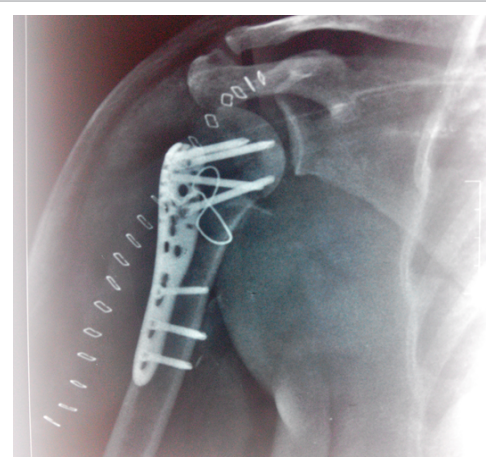

Figure 2: Post-operative x-rays after primary fixation showing the use of locking plate and stainless steel wire loop (Done outside our Institution).

fixation of fracture dislocation of right shoulder, outside our institution. Initially, she had sustained injury in a roadside accident (Figure 1). She was operated five days after the injury by a well-trained senior orthopaedic surgeon and the fracture was stabilized with Proximal Humerus Internal Locking System (PHILOS) plate and a stainless steel wire (Synthes) (Figure 2). Her shoulder was immobilized for 2 weeks. Post operatively, she complained of serous discharge coming from lower end of the surgical wound. Culture of the discharge was done 3 times, which turned out to be sterile on all occasions. She underwent supervised physiotherapy and the painless range of motion at 3 months was: flexion 0-30 degrees, extension 0-30 degrees, no active abduction possible but passive abduction possible up to 90 degrees. Due to continuing discharge from surgical site, the primary surgeon performed a surgical debridement of the wound at 3 months, where removal of loose loop of stainless steel wire was also done. After the second surgery, pain gradually increased and range of motion gradually decreased over time. At 5 months, she was referred to us with complaint of extreme pain in the shoulder, even when she attempted to change her body posture in the bed. On clinical examination there was obvious

*Corresponding author: Ravi Kumar Gupta, Government Medical College Hospital, Sector 32, Chandigarh, 160030, Punjab, India, Tel: +919646121592; E-mail: ravikgupta2000@yahoo.com

Received August 26, 2015; Accepted September 19, 2015; Published September 28,2015

Citation: Gupta RK, Malhotra A, Kumar P, Masih GD (2015) PHILOS Plate: Is it the Panacea for all Proximal Humerus Fractures? J Trauma Treat 4: 262. doi:10.4172/2167-1222.1000262

Copyright: $\odot 2015$ Gupta RK, et al. This is an open-access article distributed under the terms of the Creative Commons Attribution License, which permits unrestricted use, distribution, and reproduction in any medium, provided the original author and source are credited. 


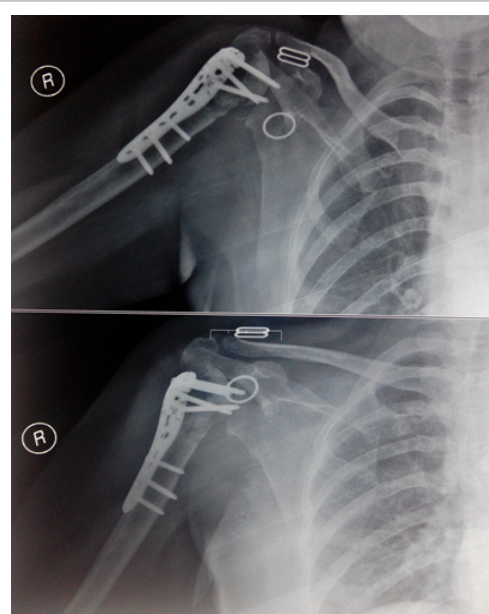

Figure 3: X-rays showing migration of locking screws into the joint.

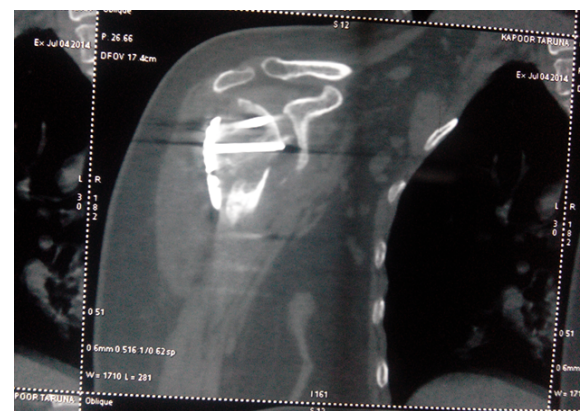

Figure 4: CT image showing non-union and intra-articular protrusion of screws.

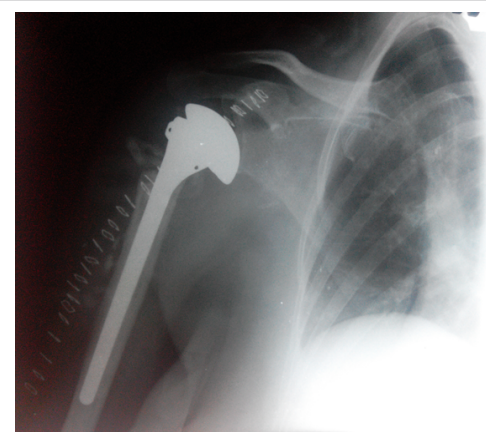

Figure 5: Post-operative Xrays after hemiarthroplasty of the shoulder.

muscle wasting over right shoulder. There was global restriction of movements, with extremely painful movements.

$\mathrm{X}$-rays of the proximal humerus showed intra-articular migration of the head screws and resorption of head; the fracture appeared to be un-united as well (Figure 3). Post operative serial check X-rays at different times were studied, which revealed gradual migration of screws into the joint (screw perforation). Nonunion and intra-articular migration of the screws was further confirmed on CT scan images (Figure 4). Glenoid articular surface was, however, found to be well preserved. Patient was planned for plate removal and hemiarthroplasty of right shoulder.

Shoulder joint was exposed with delto-pectoral approach, using the scar mark of previous surgery's incision. Scarred tissue was found during dissection. Rotator interval and rotator cuff were scarred and adherent to soft tissues and bone. Reconstruction of the rotator interval and soft tissue release of the rotator cuff was performed. Plate and screws were removed. Head and tuberosities were found resorbed with only shell of the articular surface remaining. Cemented shoulder hemiarthroplasty was performed and rotator cuff attached to prosthesis with Ethibond No. 5 suture (Figure 5). Post operatively, shoulder was immobilized with Universal shoulder immobilizer.

No local antibiotic beads were placed during the surgery. Postoperative period was uneventful with no evidence of infection throughout. She underwent a supervised physiotherapy program in the post-operative period, which consisted of passive range of motion exercises, active range of motion exercises, pendulum exercises and muscle strengthening exercises. At 12 months post-operative interval, active range of motion at shoulder joint was: flexion upto 80 degrees, abduction up to 90 degrees, external rotation up to 10 degrees and internal rotation up to 25 degrees.

\section{Discussion}

Optimal treatment of three and four-part fractures of the proximal part of humerus in patients with poor bone quality is controversial. Open reduction and internal fixation of these fractures with standard implants has been discouraged. Locked plates, which maintain angular stability in the face of axial load, have been found to provide substantial benefit in biomechanical studies, but their clinical utility has not been widely accepted. Their clinical benefit in the treatment of two-part fractures has been established, but the overall complication rate is substantial, and when they have been used for more complex fracture patterns, loss of fixation and screw perforation of the humeral head have been challenging complications [1]. Since, elderly patients with osteoporotic bones have been reported to have a greater incidence of plate-related complications, some surgeons still feel hemiarthroplasty to be a better option as a primary surgery in three and four part proximal humerus fractures in such patients [6-8].

The locking mechanism of the screws gives the surgeon an inadequate evaluation of the bone quality and a false sense of highsecurity regarding the screw fixation in the osseous fragments, even if the purchase of the screw in the cancellous bone is suboptimal, thus leading to a high rate of screw cutout (13.7\%) [2]. In the present case, we feel that the slow union at the fracture site resulted in the collapse at fracture site. The locked heads of the screws in the well-fixed plate did not allow the distal migration of the screws on the plate side, and the screws migrated into the joint by cutting the articular surface. This gave rise to the need for a secondary hemiarthroplasty of the shoulder in this case. Hemiarthroplasty in chronic cases has its own set of problems, related to increased degeneration of rotator cuff, extensive soft tissue scarring and hence impaired shoulder function. The functional outcome has been observed to be inversely proportional to the time between injury and hemiarthroplasty. Hence, it is recommended that prosthetic humeral head replacement be performed as early as possible, especially in elderly people with osteoporotic bones [9]. Mechanical stability of the implant is of utmost importance in fixing Neer 3 part fractures. Hence, locking plates take precedence in such cases because of their superior biomechanical properties as compared to traditional plates. However, while managing Neer 4 part fractures, fracture dislocations and head splitting fractures, both mechanical stability of the implant and vascularity of head become areas of concern. In addition, osteoporosis and poor bone quality further weaken the case of PHILOS plating in such situations. Hence, fracture fixation with a locking plate for a fracture dislocation of shoulder joint in a 60 year old 
Citation: Gupta RK, Malhotra A, Kumar P, Masih GD (2015) PHILOS Plate: Is it the Panacea for all Proximal Humerus Fractures? J Trauma Treat 4: 262. doi:10.4172/2167-1222.1000262

Page 3 of 3

lady with osteoporosis is a recipe for failure. We recommend a primary hemiarthroplasty of the shoulder in such a case.

\section{Conclusion}

PHILOS plate is not the panacea for all the proximal humerus fractures. While taking a decision to operate, the surgeons should be aware of the potential complications of the chosen surgical modalities, and should weigh the benefits and risks of all the available options. There is a considerable risk of osteonecrosis associated with 4 part fractures, fracture dislocations or head splitting fractures, which is not addressed by locking plates. In addition, a secondary hemiarthroplasty has its own set of problems, with impaired shoulder function being the most important one. All these arguments shift the balance in favour of a primary hemiarthroplasty in elderly patients suffering from 4 part fractures, fracture dislocations or head splitting fractures of proximal humerus.

\section{References}

1. Solberg BD, Moon CN, Franco DP, Paiement GD (2009) Surgical treatment of three and four-part proximal humeral fractures. J Bone Joint Surg Am 91: 1689-1697.

2. Clavert P, Adam P, Bevort A, Bonnomet F, Kempf JF (2010) Pitfalls and complications with locking plate for proximal humerus fracture. J Shoulder Elbow Surg 19: 489-494.

3. Lever JP, Aksenov SA, Zdero R, Ahn H, McKee MD, et al. (2008) Biomechanical analysis of plate osteosynthesis systems for proximal humérus fractures. J Orthop Trauma 22: 23-29.

4. Sommer C, Gautier E, Muller M, Helfet D, Wagner M (2003) First clinical results of the locking compression plate (LCP). Injury 34: B43-54.

5. Agudelo J, Schurmann M, Stahel P, Helwig P, Morgan S, et al. (2007). Analysis of efficacy and failure in proximal humerus fractures treated with locking plates. J Orthop Trauma 21: 676-681.

6. Christoforakis JJ, Kontakis GM, Katonis PG, Stergiopoulos K, Hadjipavlou AG (2004) Shoulder hemiarthroplasty in the management of humeral head fractures. Acta Orthop Belg 70: 214-225.

7. Brunner F, Sommer C, Bahrs C, Heuwinkel R, Hafner C, et al. (2009) Open reduction and internal fixation of proximal humerus fractures using a proximal humeral locked plate: a prospective multicenter analysis. J Orthop Trauma 23 163-172.

8. Gregory TM, Vandenbussche E, Augereau B (2013) Surgical treatment of three and four- part proximal humeral fractures. Orthopaedics \& Traumatology: Surgery \& Research 99: S197-S207.

9. Bosch U, Skutek M, Fremerey RW, Tscherne H (1998) Outcome after primary and secondary hemiarthroplasty in elderly patients with fractures of the proximal humerus. J Shoulder Elbow Surg 7: 479-484. 